\title{
Evaluation of cloud 3D printing order task execution based on the AHP-TOPSIS optimal set algorithm and the Baldwin effect
}

\author{
Chenglei ZHANG ${ }^{1,2,3 *}$, Cunshan ZHANG ${ }^{1}$,Jiaojiao ZHUANG ${ }^{2}$, Hu HA², Bo YUAH ${ }^{4}$, Jiajia LIU ${ }^{2 *}$,Shenle ZHUANG ${ }^{3}$, \\ Ronglan $\mathrm{LI}^{3}$
}

1 School of Electrical and Electronic Engineering, Shandong University of Technology, zibo, 255000, China; zhangchenglei@lyu.edu.cn

2 School of Mechanical \& Vehicle Engineering, LINYI University, linyi, 276000, China

3 Shandong Longli Electronic Co., Ltd, linyi, 276000 China

4 School of Mechanical and Electrical Engineering, Wuhan City Polytechnic, Wuhan, 430064, China

* Correspondence: zhangchenglei@lyu.edu.cn; Tel.:+86 15964819699

liujiajia@lyu.edu.cn;Tel.:+86 18753989976

\begin{abstract}
Focusing on service control factors, rapid changes in manufacturing environments, the difficulty of resource allocation evaluation, resource optimization for 3D printing services (3DPSs) in cloud manufacturing environments and so on, an indicator evaluation framework is proposed for the cloud 3D printing (C3DP) order task execution process based on a Pareto optimal set algorithm that is optimized and evaluated for remotely distributed 3D printing equipment resources. Combined with the multi-objective method of data normalization, an optimization model for C3DP order execution based on the Pareto optimal set algorithm is constructed with these agents' dynamic autonomy and distributed processing. This model can perform functions such as automatic matching and optimization of candidate services, and it is dynamic and reliable in the C3DP order task execution process based on the Pareto optimal set algorithm. Finally, a case study is designed to test the applicability and effectiveness of the C3DP order task execution process based on the analytic hierarchy process and technique for order of preference by similarity to ideal solution (AHP-TOPSIS) optimal set algorithm and the Baldwin effect.
\end{abstract}

Keywords: Cloud Manufacturing(CMfg); 3D Printing Device Resources; HPSO; Muti-objective Optimization; Baldwin effect

\section{Introduction}

Intelligent algorithms are the most commonly used tool to solve NP-complete combination optimization problems. After years of development, many different random search strategies have emerged[1]. They all form their own iterative search mechanisms by simulating the behavior and evolution of natural ecology. They are simple, universal, robust and easy to use in parallel processing[2]. To solve the problem of computing resource allocation in a cloud 3D printing service (C3DPS) resource pool, it is necessary to consider both the constraints of the task graph and the nodes. In the neighborhood search algorithm, the typical simulated annealing and tabu search algorithms are used due to the strong randomness and the need for only a single iteration based on an individual search. There is a very low probability of finding an optimal solution within the feasible solution set during a short iteration period. Presently, this algorithm is not suitable for solving combination optimization problems such as scheduling when combined with another algorithm application[3]. However, only the heredity and immunity algorithms 
show good performance in various applications and other improvements in evolutionary learning algorithms. Others, such as artificial neural network and DNA calculation algorithms, are less mature in the computational process of solving problems in mechanics as applied to combination optimization. In addition, the ant colony algorithm and particle swarm optimization (PSO) algorithm based on the swarm intelligence algorithm are the most widely used in scheduling problems. However, the self-learning particle swarm algorithm is designed for continuous-type variable numerical value optimization problems. To a certain extent, most of the improved algorithms convert the original numerical changes into individuals in a particle swarm, and the optimum crosscurrent operation or sub-optimal exchange has no obvious practical application. The optimum crosscurrent operation is also called genetic PSO[4].

The evaluation of C3DPS quality belongs to the field of multiple-criteria decision-making (MCDM)[5]. A study of relevant data around the world reveals that research on service quality is primarily based on scientificity, reliability, a combination of quantitative and qualitative aspects, and other principles. Therefore, there are three primary methods for comprehensively evaluating C3DPS quality: the classical comprehensive evaluation method of cloud services (CSs), the CS evaluation method based on multi-attribute decision-making and the quality of service (QoS)[6,7]. These methods are used in the analytic hierarchy process (AHP), fuzzy mathematics, the effectiveness function, principal component analysis (PCA), probability density functions (PDFs), the technique for order of preference by similarity to ideal solution (TOPSIS), data envelopment analysis (DEA), the Pareto optimal set algorithm and other methods[8]. Among these methods, the AHP method is a multi-objective decision analysis method combined with qualitative and quantitative analysis. It is primarily applied in uncertain situations with multiple evaluation criteria. Fuzzy mathematics is a mathematical theory and method used to study and manage the phenomenon of fuzziness. The utility function method is usually employed to represent the relationship between the utility obtained by the consumer in consumption and the quantity of the product portfolio[9]. The PCA is a statistical method. It attempts to replace the original index by recombining the original correlations (such as P indicators) into a new set of unrelated and interrelated indicators. The value of the PDF at any given point in the set of possible values taken by the random variable can be interpreted as providing the relative likelihood that the value of the random variable will equal that sample. The TOPSIS method sorts potential solutions according to the closeness among a limited number of negative ideal solutions and the preference by similarity to ideal solution (PSIS), which is a comparison of the relative advantages and disadvantages of existing objects[10]. The DEA method is a quantitative analysis method that can input and output several indicators. It uses linear programming to achieve a relative validity evaluation of the amendment of a decision-making unit. This method cannot sort all the decision-making targets[11].

Multi-attribute decision-making combination evaluation methods include the AHP method, fuzzy mathematics, the effectiveness function, the PCA method and the PDF method[12]. These methods have the advantage of providing a single comprehensive evaluation of qualitative analysis and quantitative analysis, and they overcome the defects of their individual components. However, the disadvantage is that most of the evaluation indicators are qualitative analyses, and the number of indicators is undesirably large. The TOPSIS method is suitable for small restrictions on the number and types of evaluation indicators. It can be objectively optimized for several decision-making options[13]. The Pareto optimal set algorithm has the advantages of simplicity, good convergence and fast search speed. It is applied in cloud-service evaluation; it is efficient and makes it relatively easy to obtain satisfactory evaluation results. In summary, the Pareto optimal set intelligent optimization algorithm retains the advantages and addresses the disadvantages of the improved local optimal solution or global optimal solution algo- 
rithm; its use in solving the resource allocation problem is relatively suitable, and it has potential for solving the resource allocation problem.

The research on index systems is mainly divided into the reputation evaluation index, QoS evaluation index, service resource combination evaluation index and other evaluation indexes[14]. According to the characteristics of the evaluation index, it is a cloud service evaluation method for multi-attribute decision-making. This method puts fewer restrictions on the characteristics of the evaluation index and the type of index and can optimize multiple decision-making schemes. An intelligent optimization algorithm has a simple algorithmic principle. The convergence and search speed are good, the evaluation efficiency is high, it is relatively easy to obtain satisfactory evaluation results, and the related research fields are expanding. Therefore, this paper proposes a multi-attribute decision service evaluation method based on an intelligent optimization algorithm, that is, a multi-attribute decision evaluation method based on a Pareto optimal set algorithm. To solve the problem of computing resource allocation, it is necessary to consider the constraints of both the task graph edges and nodes in the solution process of the C3DPS resource pool[15]. Among neighbourhood search algorithms, typical simulated annealing and abut search have strong randomness and use only a single individual based on the iterative search, so the probability of finding a feasible solution is extremely low in a short time (iteration time). At present, they are mostly combined with other algorithms, but these are not suitable for solving optimization problems such as scheduling combinations.

Among evolutionary learning algorithms, only immune algorithms and genetic algorithms have been proven to show good performance in various applications and improvements. Other algorithms are less developed or more immature in the neighbourhood of optimization, such as artificial neural networks and DNA computing. In addition, the ant colony algorithm and particle swarm algorithm are widely used in scheduling problems. However, the self-learning mechanism of PSO was originally designed for continuous numerical optimization problems[16]. For discrete combination optimization problems, the speed and position learning mechanisms of these algorithms are not suitable. Most of the improvements convert the original numerical change into a crossover operation or exchange sub-operation between an individual and the current optimal solution. To a certain extent, this method is transformed into a genetic particle swarm algorithm, and the effect is not obvious in practical applications. In summary, the intelligent optimization algorithm for a Pareto optimal set is based on the above-mentioned advantages and disadvantages[17]. It is a newly improved local optimal solution or global optimal solution algorithm that is relatively suitable and has great potential for solving the problem of computing resource allocation.

\section{A framework for cloud service evaluation based on a hybrid multi-objective BM-MOPSO evaluation model}

The Materials and Methods should be described with sufficient details to allow others to replicate and build on the published results. Please note that the publication of your manuscript implicates that you must make all materials, data, computer code, and protocols associated with the publication available to readers[18]. Please disclose at the submission stage any restrictions on the availability of materials or information. New methods and protocols should be described in detail while well-established methods can be briefly described and appropriately cited.

Research manuscripts reporting large datasets that are deposited in a publicly available database should specify where the data have been deposited and provide the relevant accession numbers. If the accession numbers have not yet been obtained at the time of submission, please state that they will be provided during review. They must be provided prior to publication[19]. Interventionary studies involving animals or humans, 
and other studies that require ethical approval, must list the authority that provided approval and the corresponding ethical approval code.

Based on Pareto optimality theory and the AHP-TOPSIS evaluation model, this framework is used to objectively determine the weight of each evaluation set and indicator by the AHP method based on MATLAB software, and then the TOPSIS method is used to analyse the closeness of the optimal solution and determine the overall similarity between an optimal and ideal solution[20]. Therefore, it is possible to construct a new AHP-TOPSIS evaluation ideal value approximation model for decision-making. Based on this thesis, this paper constructs a new AHP-TOPSIS judgment approaching the ideal solution, which can be used in decision-making.

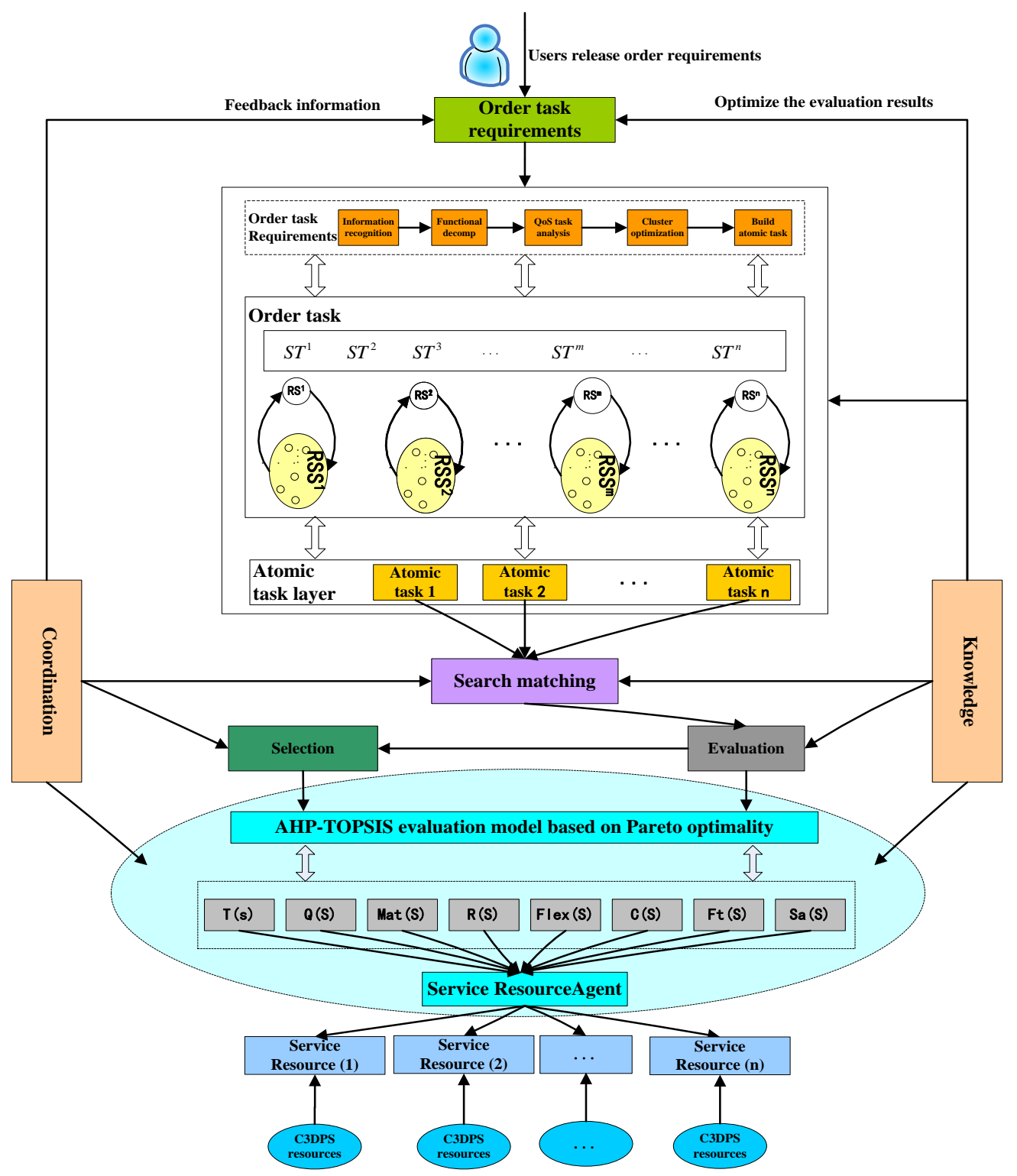

Figure 1. Framework of the evaluation method of the C3DPS order task execution process based on the AHP-TOPSIS optimal set algorithm and Baldwin effect.

One approach to cloud service evaluation is as follows: In an intelligent C3DPS platform, task-order requirements are normalized descriptions given in detail by task-order requirements.Task decomposition calls a parsing function and an analysis task that forms a one-to-one atomic task[21]. According to the atomic task, search matching is used to perform supply and demand matching of service resources, form a dual feasible 
solution for the C3DPS resource candidate service set, and provide feedback to users. According to the optimization of user requirements, it then calls a Pareto optimal intelligent optimization algorithm based on the C3DPS platform. This algorithm is optimized to objectively determine the weight of each evaluation set and indicator by the AHP method and construct a comprehensive AHP-TOPSIS evaluation model based on a Pareto-optimal set. Finally, it performs Pareto-optimal evolutionary computations and many local search strategies within the multi-objective evolutionary method. Comprehensive superiority is calculated as an evaluation indicator in this cloud service evaluation method, and it can also be used in combining and optimizing design schemes. Therefore, the optimal service resource is called. The framework of the evaluation method of the C3DPS order task execution process based on the AHP-TOPSIS optimal set algorithm and Baldwin effect is shown in Figure 1:

\section{Intelligent optimization algorithm for the Pareto optimal set and AHP}

\subsection{Intelligent optimization algorithm for Pareto optima}

The Pareto optimal condition is as follows: In the process of information resource allocation, the marginal rate of technical substitution of two kinds of information resource $\mathrm{X}$ and $\mathrm{Y}$ in any computer resource is equal to the marginal rate of technical substitution of producing these two kinds of information resources $X$ and $Y$; that is,

$$
M R T_{X Y}=M R S_{X Y}^{A}
$$

where $M R T$ is an ideal state of a computing resource allocation and $M R S_{X Y}^{A}$ is a marginal rate of technical substitution of the two kinds of information resource $X$ and $Y$ in any computing resource.

The intelligent optimization algorithm for Pareto optima is derived from the above concept[23]. Based on the analysis of the existing intelligent optimization search strategies, a multi-objective optimization algorithm for Pareto optimal and AHP-TOPSIS evaluation models is proposed. It is a multi-objective evolutionary algorithm that combines evolutionary computation and a multi-local search strategy.

Definition: Assuming that is the outer set in the th generation, each individual in is assigned an intensity value, namely:

$$
S_{i}^{(k)}=\frac{t_{i}^{(k)}}{n^{(k)}+1} \quad i=1,2 \ldots, n_{T}^{(k)},
$$

where, $t_{i}^{(k)}=\left\{\left(u \mid u_{i}^{(k)}<u\right.\right.$ s.t. $\left.u_{i}^{(k)} \in T^{(k)}\right\} \mid, T^{(k)}=D o m S^{(k)} \cup T^{(k-1)}, n_{T}^{(k)}$ is the size of set $T^{(k)}$, and $t_{i}^{(k)}$ is the number of individuals who dominate the set.

A strength value is assigned such that each individual can be computed and multi-locally searched for the evolution of the Pareto optimal set intelligent optimization algorithm, and this value is the fitness determined by its advantages and disadvantages, namely:

$$
f_{i}^{(k)}=1+\sum_{u_{j} \in T^{(k)} \wedge u_{j} \wedge u_{i}} S_{i}^{(k)},
$$

Here, the fitness of an individual is equal to the sum of all the external individuals that dominate it. In addition, the size of the set is chosen so that the individual is infinitely close to the fitness value. 


\subsection{Analytic hierarchy process}

In the 1970s, Professor T. L. Saaty, an American operational research scientist, proposed a multi-objective decision analysis method called the AHP, which is mainly applied to decision-making problems under multiple evaluation criteria[24].

The modelling steps of the AHP are as follows:

(1) Establish a hierarchical structure model

The hierarchical structure model is decomposed into the various factors that are contained in the problem and form several levels from top to bottom according to different attributes[25]. The structure model framework includes the following levels: the highest level (the overall goal of the complex system); the middle level (the planning and decision-making, the measures and adopted policies, and the criteria for achieving the goals); and the lowest level (various strategies and constraints).

Definition: Assuming that the set of evaluation indicators is $T=\left\{t_{1}, t_{2}, \ldots, t_{n}\right\}$, the evaluation indicators of each factor are compared with a pair of importance degrees, and the determined value $\alpha_{i j}$ is set to represent the importance degree of $t_{i}$. Then, the hierarchical structure model is as follows:

$$
\alpha_{i j}>0, \alpha_{i j}=1 / \alpha_{j i}
$$

where $\alpha_{i j}$ is the scale.

Then, the judgment matrix $\mathrm{D}$ is as follows:

$$
D=\left[\begin{array}{cccc}
X_{11} & X_{12} & \cdots & X_{1 n} \\
X_{21} & X_{22} & \cdots & X_{2 n} \\
\vdots & \vdots & \vdots & \vdots \\
X_{m 1} & X_{m 2} & \cdots & X_{m n}
\end{array}\right]=\left[\begin{array}{cccc}
\frac{X_{1}}{X_{1}} & \frac{X_{1}}{X_{2}} & \cdots & \frac{X_{1}}{X_{n}} \\
\frac{X_{2}}{X_{1}} & \frac{X_{2}}{X_{2}} & \cdots & \frac{X_{2}}{X_{n}} \\
\vdots & \vdots & \vdots & \vdots \\
\frac{X_{n}}{X_{1}} & \frac{X_{n}}{X_{2}} & \cdots & \frac{X_{n}}{X_{n}}
\end{array}\right],
$$

It can be seen from the above that for the positive definite reciprocal judgment matrix $D$, the maximum characteristic root $\lambda_{\max }$ exists and is unique, and the weight $w_{i}$ is composed of positive elements of vectors and is unique.

(2) Construct a judgment matrix

The multi-level system is divided into several hierarchical levels according to different goals and functions[26]. Among them, the judgment matrix is constructed with the pairwise comparison method and comparison scale at the lowest level.

The elements in each row of the judgment matrix are multiplied as follows:

$$
M_{i}=\prod_{i=1}^{n} \alpha_{i j}
$$

$M_{i}$ is calculated by taking the n-th power root:

$$
W_{i}=\sqrt[n]{M_{i}}
$$


$W_{i}$ in the vector is normalized:

$$
W_{i}=W_{i} / \sum_{i=1}^{n} W_{i},
$$

Here, the values of vector $W_{i}$ are normalized for the consistency check.

(3) Determine the hierarchical order and perform a consistency check

Here, the method determines the correlation degree between adjacent level elements in the above judgment matrix. Through the construction of two comparison judgment matrices and the mathematical method of matrix operations, the importance order of the related elements is determined for a certain element in the previous level.

Definition: In checking the consistency of the judgment matrix, knowledge and experience can meet different conditions. The specific formula is as follows:

$$
C_{R}=\frac{C_{i}}{R_{i}}=\frac{\lambda_{\max }-n}{(n-1) R_{i}}
$$

where $C_{i}$ is the indicator of the consistency check, $n$ is the order of the judgment matrix, and $R_{i}$ is the average value of the consistency check.

(4) Determine the hierarchical total ranking and perform a consistency check

The combination weight vector of each layer element is calculated and ranked by the formula of the combination consistency check. Therefore, it determines the importance degree of each element at the bottom of the hierarchical structure model. In the traditional AHP method, it is difficult to verify the consistency of the judgment matrix, and this matrix is greatly affected by expert knowledge and preferences, which limits its promotion and application[27]. The concept of multi-indicator evaluated entropy is proposed, and the indicator weight solution method solves the problems of the traditional AHP method. The evaluation entropy refers to the confusion degree of the evaluation value of each candidate C3DPS set in a comprehensive evaluation.

Definition: Assuming that for a certain service request, the set of candidate C3DPSs is $C S=\left(S_{1}, S_{2}, \ldots, S_{n}\right)$ and that $Q T_{11}, Q T_{12}, \ldots, Q T_{1 n}$ are defined as the evaluation values of the candidate C3DPSs on the indicators, the evaluation entropy of the indicators $Q T_{1}$ is:

$$
\operatorname{Diff}\left(Q T_{1}\right)=\frac{\sqrt{\frac{\sum_{i=1}^{\mathrm{n}}\left(Q T_{1 i}-\overline{Q T_{1}}\right)^{2}}{n-1}}}{Q T_{1}}
$$

The solution formula for index evaluation entropy is introduced into other secondary indicators of the QoS index, and $\operatorname{Diff}\left(Q T_{2}\right), \ldots, \operatorname{Diff}\left(Q T_{\mathrm{i}}\right)$ are obtained; then, the relative weight corresponding to $Q T_{x}(x=1,2, \ldots, n)$ is: 


$$
W\left(Q T_{\mathrm{x}}\right)=\frac{\operatorname{Diff}\left(Q T_{x}\right)}{\sum_{i=1}^{n} \operatorname{Diff}\left(Q T_{x}\right)}
$$

where $W\left(Q T_{\mathrm{x}}\right)$ is the weight value, $\operatorname{Diff}\left(Q T_{x}\right)$ is the indicator evaluation entropy of $\operatorname{Diff}\left(Q T_{x}\right)$, and $\sum_{i=1}^{n} \operatorname{Diff}\left(Q T_{x}\right)$ is the sum of the entropy values of $Q T_{x}(x=1,2, \ldots, n)$.

Based on Pareto optimal theory, the improved AHP-TOPSIS evaluation model is used to objectively determine the weight of each evaluation set and index[28]. The closeness of the evaluation model is determined by analyzing and making decisions to obtain the optimal solution that brings about an overall similarity between the best alternative and the ideal scheme combined with the TOPSIS optimization method.

\section{Mathematical model of C3DPS order task execution evaluation based on the AHP-TOPSIS evaluation model}

In the process of multi-attribute decision-making, the weight of each attribute reflects the relative importance of the attributes, which directly affects the result of decision-making. Therefore, the weight of each attribute is one of the key issues in multi-attribute decision-making that determines the results of decision-making. Aiming to solve a multi-attribute decision-making problem in which the attribute weight information is determined and the attribute value is an intuitionistic fuzzy number, a decision analysis method for the AHP-TOPSIS evaluation model is proposed. This analysis method is widely used in multi-attribute decision-making problems. The AHP-TOPSIS comprehensive evaluation model is a comprehensive evaluation and optimization method that combines the AHP and TOPSIS. The weight vector of each evaluation indicator is objectively determined and calculated to achieve comprehensive superiority by this evaluation model[29]. The basic principle of TOPSIS is to sort the evaluation objects by the distance between the fuzzy positive ideal solution and its similarity to fuzzy comprehensive attributes in the multi-objective decision-making problem.

\subsection{Establish an initial evaluation matrix $A$}

Definition: Suppose that a set $A=\left\{A_{1}, A_{2}, \ldots, A_{m}\right\}$ of schemes is composed of $\mathrm{m}$ schemes $A_{1}, A_{2}, \ldots, A_{m}$ and that each scheme also corresponds to a number of evaluation indicators $X_{1}, X_{2}, \ldots, X_{n}$. The set of evaluation indicators is $X=\left\{X_{1}, X_{2}, \ldots, X_{n}\right\}$. Then, the initial evaluation indicator matrix can be expressed as:

$$
A=\left(X_{i j}\right)_{m \times n}=\left[\begin{array}{cccc}
X_{11} & X_{12} & \cdots & X_{1 n} \\
X_{21} & X_{22} & \cdots & X_{2 n} \\
\vdots & \vdots & \vdots & \vdots \\
X_{m 1} & X_{m 2} & \cdots & X_{m n}
\end{array}\right]
$$

where $X_{i j}$ is the $j$-th evaluation indicator in the $j$-th scheme.

\subsection{Establish a weighted standardized decision matrix}

The evaluation indicators can be divided into two categories: consumption indicators and profit indicators. The higher the profit indicator is, the lower the consumption indicator. These are two kinds of indicators that can also be divided into measurement indicators and non-measurement indicators with different dimensions and dimensional units[30]. On this basis, it is necessary to strictly define the meaning of indicators and 
provide reference standards, which will eliminate the incontestability of the resulting indicators and carry out dimension normalization of the evaluation indicators in the evaluation of non-metric indicators.

The standardized decision calculation method for the initial evaluation matrix is described below.

(1) The consumption indicator is

$$
b_{i j}=\frac{X_{i j}-\min _{j}\left(X_{i j}\right)}{\max _{j}\left(X_{i j}\right)-\min _{j}\left(X_{i j}\right)},
$$

where $X_{i j}-\min _{j}\left(X_{i j}\right)$ is the difference between the evaluation indicator value and the minimum evaluation indicator value in the initial evaluation matrix; $\max _{j}\left(X_{i j}\right)-\min _{j}\left(X_{i j}\right)$ is the difference between the maximum value and the minimum value in the initial evaluation matrix.

(2) The profit indicator is

$$
b_{i j}=\frac{\max _{j}\left(X_{i j}\right)-X_{i j}}{\max _{j}\left(X_{i j}\right)-\min _{j}\left(X_{i j}\right)},
$$

where $\max _{j}\left(X_{i j}\right)-X_{i j}$ is the difference between the maximum evaluation indicator value and the evaluation indicator value in the initial evaluation matrix, and $\max _{j}\left(X_{i j}\right)-\min _{j}\left(X_{i j}\right)$ is the difference between the maximum value and the minimum value in the initial evaluation matrix.

According to the life cycle evaluation indicator system of C3DPSs, a standardized decision matrix for multi-attribute decision-making is constructed. According to the accumulation process of the performance indicator $M O p t(T, Q, M a t, R, R l$, Flex, $\mathrm{C}, \mathrm{Ft}, \mathrm{SF}, \mathrm{Sa}$ ), the objective function of multi-objective optimization is determined, and the performance of the C3DPSs can be evaluated through four sub-evaluation indicator systems[31]. Assuming that the service resources of C3DPSs are evaluated, ORS $=\left\{r_{1}, r_{2}, \ldots, r_{n}\right\}$ will be selected as a candidate set of service resources in the C3DP order execution process. A standardized decision matrix for multi-attribute decision-making is shown in Table 1.

\begin{tabular}{|c|c|c|c|c|c|c|}
\hline & $r_{1}$ & $r_{2}$ & $\ldots$ & $r_{i}$ & $\ldots$ & $r_{n}$ \\
\hline $\mathrm{T}$ & $\left(\mu_{1}^{T}, v_{1}^{T}\right)$ & $\left(\mu_{2}^{T}, v_{2}^{T}\right)$ & $\ldots$ & $\left(\mu_{i}^{T}, v_{i}^{T}\right)$ & $\ldots$ & $\left(\mu_{n}^{T}, v_{n}^{T}\right)$ \\
\hline Q & $\left(\mu_{1}^{Q}, v_{1}^{Q}\right)$ & $\left(\mu_{2}^{Q}, v_{2}^{Q}\right)$ & $\ldots$ & $\left(\mu_{i}^{Q}, v_{i}^{Q}\right)$ & $\ldots$ & $\left(\mu_{n}^{Q}, v_{n}^{Q}\right)$ \\
\hline Mat & $\left(\mu_{1}^{M a t}, v_{1}^{M a t}\right)$ & $\left(\mu_{2}^{M a t}, v_{2}^{M a t}\right)$ & $\ldots$ & $\left(\mu_{i}^{\text {Mat }}, v_{i}^{\text {Mat }}\right)$ & $\ldots$ & $\left(\mu_{n}^{\text {Mat }}, v_{n}^{\text {Mat }}\right)$ \\
\hline $\mathrm{R}$ & $\left(\mu_{1}^{R}, v_{1}^{R}\right)$ & $\left(\mu_{2}^{R}, v_{2}^{R}\right)$ & $\ldots$ & $\left(\mu_{i}^{R}, v_{i}^{R}\right)$ & $\ldots$ & $\left(\mu_{n}^{R}, v_{n}^{R}\right)$ \\
\hline $\mathrm{Rl}$ & $\left(\mu_{1}^{R l}, v_{1}^{R l}\right)$ & $\left(\mu_{2}^{R l}, v_{2}^{R l}\right)$ & $\ldots$ & $\left(\mu_{i}^{R l}, v_{i}^{R l}\right)$ & $\ldots$ & $\left(\mu_{n}^{R l}, v_{n}^{R l}\right)$ \\
\hline Flex & $\left(\mu_{1}^{F l e x}, v_{1}^{F l e x}\right)$ & $\left(\mu_{2}^{F l e x}, \nu_{2}^{F l e x}\right)$ & $\ldots$ & $\left(\mu_{i}^{F l e x}, v_{i}^{F l e x}\right)$ & $\ldots$ & $\left(\mu_{n}^{F l e x}, v_{n}^{F l e x}\right)$ \\
\hline
\end{tabular}

Table 1. Standardized decision matrix for multi-attribute decision-making. 
The evaluation matrix is constructed from the proximity analysis of the TOPSIS method, and the result vector $F$ of the AHP-TOPSIS comprehensive evaluation is as follows:

$$
F=W \times B
$$

In the formula, $B$ is an evaluation matrix formed from the closeness value of each evaluation object, and $W$ is the weight calculated by the analytic hierarchy process.

\section{The solution of the C3DPS quality evaluation model}

4.1. Hybrid multi-objective particle swarm optimization (PSO) algorithm based on the Baldwin effect (BM-MOPSO)

BM-MOPSO is an intelligent algorithm based on multi-objective PSO that combines Baldwin's learning strategy idea, a population global target value scalar parameter, a scalar parameter for the population global objective value, intuitionistic fuzzy membership and a ranking method. In the application of the hybrid multi-objective PSO algorithm, it solves the above-mentioned C3DPS quality evaluation problem[35]. This is the key to the problem of adopting the learning of the Baldwin effect within a certain period so that the global search and learning strategies can ensure the interactive operation of the algorithm in a fixed period.

(1) Baldwin effect learning strategy

As a learning method, the Baldwin effect learning strategy can effectively reduce the selection pressure. This not only affects the characteristics of the search space but also increases the polymorphism of the genetic process and transforms the shape of the dominant search space. A local search can also be carried out based on the Baldwin effect that improves the non dominated solution of PSO[36]. The mathematical formula of this learning function is as follows:

$$
Y_{i j}=\left\{\begin{array}{c}
p_{z j}+k \times\left(p_{i j}-p_{z j}\right), \quad p_{z j}<p_{i j} \\
p_{z j}+k \times\left(p_{z j}-p_{i j}\right), \quad p_{i j}<p_{z j} \\
p_{i j}, \quad \text { Otherwise }
\end{array}\right.
$$

where $i, z=\{1,2, \ldots, m\}, m$ is the number of particles in the swarm, $\mathrm{j}=\{1,2, \ldots, n\}, n$ is the dimension of the particle swarm and is the step size of the observation interval.

If $p_{i j}$ is greater than $p_{z j}$, particles $p_{z j}$ learn from particles $p_{i j}$; if $p_{z j}$ is greater than $p_{i j}$, particles $x_{i j}$ learn from particles $x_{z j}$; if neither is dominated by the other, the particles do not learn.

(2) Local search strategy based on the BM-MOPSO algorithm

When the particle swarm performs a local search for the Baldwin effect, one of the following situations will occur:

1) When searching the initial points, most particles are far away from the Pareto frontier in the space, and it is easy to find the dominant solution in the region, which leads to high search efficiency. At a certain time, the optimal solution set of the population is sorted by Pareto dominance, and the particles are dynamically updated to the Pareto solution set. At the same time, the method learns from the dominant solution so that the particle swarm can more quickly approach the Pareto frontier. 
2) If the local search times of PSO converge to the threshold flag, the obtained solutions are all non-dominated solutions, which indicates that the population has fallen into a local extreme point at this time. The direction of the non-dominated solutions is:

$$
\mathrm{d}=\sum_{\mathrm{i}=1}^{\mathrm{flag}} \frac{\mathrm{x}_{\mathrm{i}}-\mathrm{x}_{0}}{\left\|\mathrm{x}_{\mathrm{i}}-\mathrm{x}_{0}\right\|},
$$

where $\mathrm{x}_{\mathrm{i}}(\mathrm{i}=1,2, \ldots$, flag $)$ is the $\mathrm{i}$-th non-dominated solution in the local search and $\mathrm{x}_{\mathrm{i}}-\mathrm{x}_{0}$ is the distance from the local extreme point to the initial value in the local search.

3) If the above two conditions are satisfied, then the number $d$ of $x_{i}$ and the non-dominated solution $\mathrm{q}(\mathrm{q}=3)$ satisfy one of the following conditions:

If $\mathrm{d}<\mathrm{q}$, the method learns from the dominating solution so that the particle swarm can more quickly approach the Pareto frontier.

If $\mathrm{d} \geq \mathrm{q}$, the search direction changes, and the PSO can be guided and diffused in the optimization direction.

The local search method of the BM-MOPSO algorithm is as follows:

Step 1: The parameters are initialized; that is, the initial position of PSO is $\left(\mathrm{x}_{0}, \mathrm{y}_{0}\right)$, its iteration number is $\mathrm{i}=0$, and the direction of the non-dominated solution is $\mathrm{d}=0$.

Step 2: In the initial position of $\mathrm{PSO}\left(\mathrm{x}_{0}, \mathrm{y}_{0}\right)$, a dominating solution is randomly selected and marked as $\mathrm{y}_{1}$.

Step 3: If $\mathrm{y}_{1}<\mathrm{y}_{0}$, the initial position of PSO is locally searched by the Baldwin effect learning strategy and is set to $x_{\text {new }}=y_{0}+k \times\left(y_{1}-y_{0}\right)$ (k is the number of executions of the Baldwin effect learning strategy); then, the method goes to step 5. If $\mathrm{y}_{0}<\mathrm{y}_{1}$, then the Pareto solution set is dynamically updated. At the same time, the method learns from the dominant solution. The particle swarm can move closer to the Pareto frontier, ensuring that the individual particle swarm will have this learning ability. If $\mathrm{x}_{\text {new }}=\mathrm{y}_{1}+\mathrm{k} \times\left(\mathrm{y}_{0}-\mathrm{y}_{1}\right)$, the method goes to step 5 ; otherwise, it goes to step 4 .

Step 4: If $\mathrm{d}++$, then PSO calculates the direction $\mathrm{d}$ of the non-dominated solution. If $\mathrm{d} \geq \mathrm{q}$, it reinforces learning to calculate the optimal step size $\mathrm{s}$ and explore the optimal position $\mathrm{x}_{\text {new }}=\mathrm{y}_{0}+\mathrm{d} \times \mathrm{s}$ and then goes to step 5; otherwise, it goes to step 2 .

Step 5: If $i \leq$ flag, the method goes to step 2; otherwise, this particle swarm population falls into a local extreme point, and $\mathrm{x}_{\text {new }}$ is the next initial value of particle swarm $\mathrm{x}_{0}$.

4.2. The basic process of the multi-objective particle swarm optimization algorithm based on the Baldwin effect

The basic process of multi-objective BM-MOPSO is shown in Figure 2. The algorithm steps are analyzed below. 


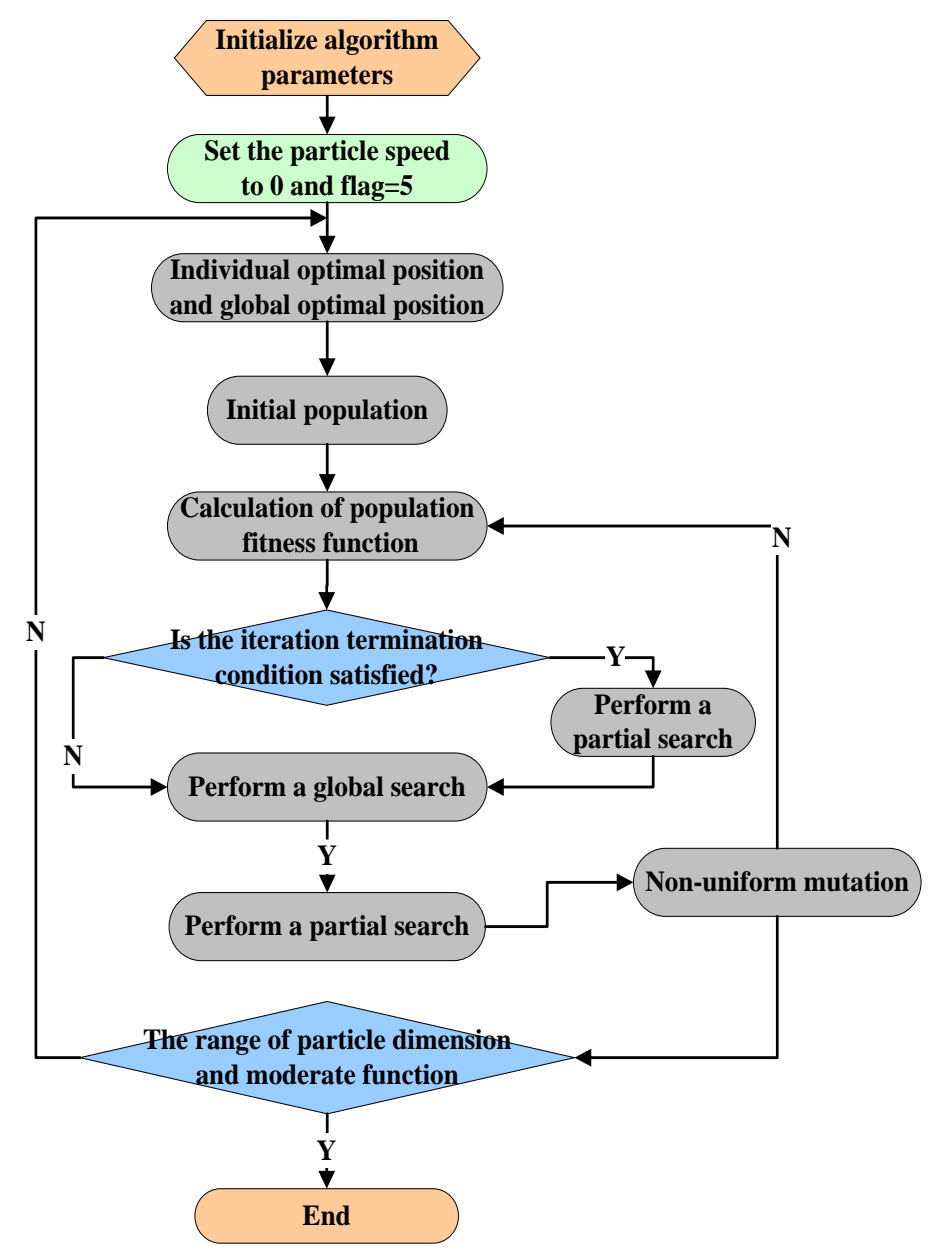

Figure 2. The basic process of the multi-objective BM-MOPSO algorithm.

(1) Particle initialization

First, the maximum number of iterations, number of independent variables of the objective function, maximum velocity of particles and position information are set randomly in the velocity interval and search space to obtain a one-to-one mapping between the service resources and particles, that is, to perform particle initialization.

Assume that each particle is an optional combination of service resources, where a service resource is selected from each candidate service set list $C R S_{i}$ to form a combination of service resources[37]. The initial scale of the particles is the $N$-population of the feasible dimension space $S=\left\{p_{1}, p_{2}, \ldots, p_{n}\right\}$, and each scale of particles corresponds to an optional combination of the number of service resources.

For example, let the combination of C3DP optional service resources be $\left\{C R S_{1}^{5}, C R S_{2}^{4}, C R S_{3}^{1}, C R S_{4}^{5}\right\}$ which means that a C3DPS resource is selected by the serial atomic task sequence $O R_{1}$ to be the fifth service resource $C R S_{1}^{5}$ in the set of service resources $C R S_{1}$. The serial atomic task sequence $O R_{2}$ is the fourth service resource $C R S_{2}^{4}$ in the set of service resources $C R S_{2}$ [38].

In the same way, the order of particles mapped one by one is . A mapping example of the composition of service resources and particles is shown in Figure 3. 


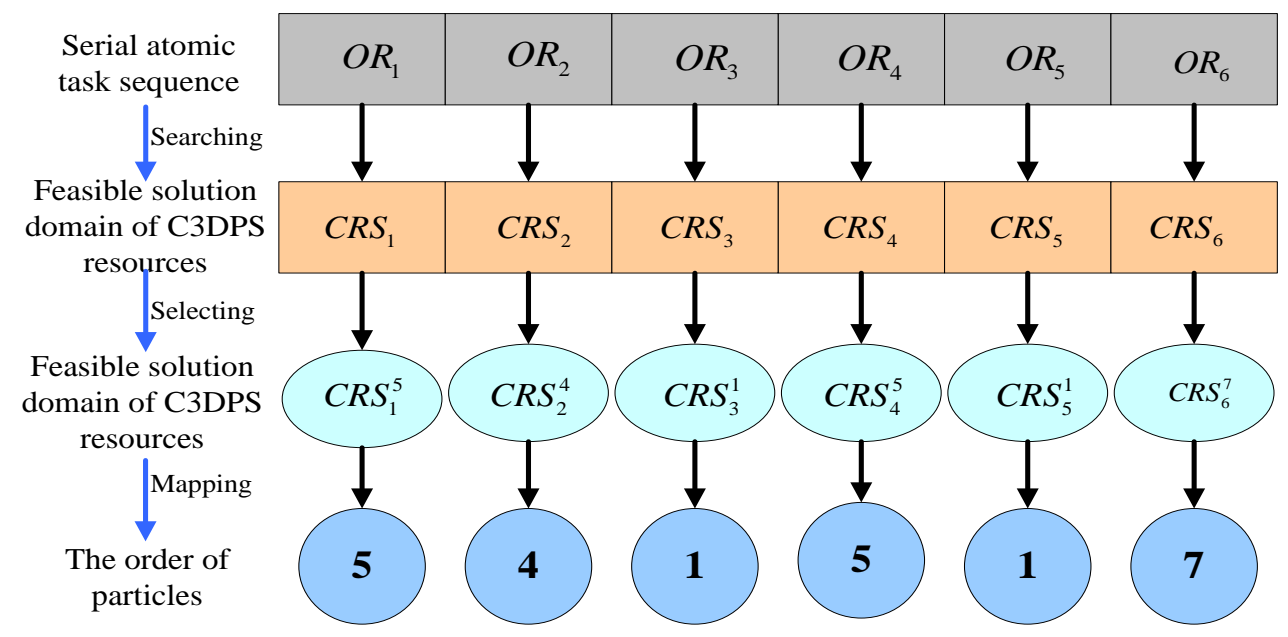

Figure 3. Mapping example of the composition of service resources and particles.

(2) Set of fitness functions

From the above objective function $M O p t(\mathrm{OR})$, the optimal value of each index set is selected by the evaluation of the C3DP order task in the feasible solution domain of the service resource candidate service subset. Therefore, the moderation function of the BM-MOPSO algorithm is calculated as follows:

$$
\begin{aligned}
& \text { fitness }\left(p^{i}\right)=w_{1}\left(\sum_{j=1}^{n} T\left(C R S_{j}^{p_{j}^{j_{j}}}\right)\right)^{-1}+w_{2}\left(\sum_{j=1}^{n} Q\left(C R S_{j}^{p_{j}^{j_{j}}}\right)\right)^{-1}+w_{3} \prod_{i=1}^{\mathrm{n}}\left(\sum_{j=1}^{n} M \mathrm{at}\left(C R S_{j}^{p_{j}^{j_{j}}}\right)\right)^{-1}+w_{4} \prod_{i=1}^{\mathrm{n}}\left(\sum_{j=1}^{n} R\left(C R S_{j}^{p_{j}^{j_{j}}}\right)\right)^{-1}, \\
& w_{5} \prod_{i=1}^{\mathrm{n}}\left(\sum_{j=1}^{n} \operatorname{Flex}\left(C R S_{j}^{p_{j}^{j_{j}}}\right)\right)^{-1}+w_{6}\left(\sum_{j=1}^{n} C\left(C R S_{j}^{p_{j}^{j_{j}}}\right)\right)^{-1}+w_{7} \prod_{i=1}^{\mathrm{n}}\left(\sum_{j=1}^{n} F \mathrm{t}\left(C R S_{j}^{p_{j}^{j_{j}}}\right)\right)^{-1}+w_{8} \prod_{i=1}^{\mathrm{n}}\left(\sum_{j=1}^{n} \operatorname{Sa}\left(C R S_{j}^{p_{j}^{j_{j}}}\right)\right)^{-1}
\end{aligned}
$$

In the formula, $w_{1}, w_{2}, w_{3}, w_{4}, w_{5}, w_{6}, w_{7}$ and $w_{8}$ are the weight ratios of C3DP equipment service resources. The larger the fitness function fitness $\left(p^{i}\right)$ is, the better the particle $p^{i}$.

(3) The range of the particle dimension and moderate function

In the BM-MOPSO algorithm, the learning strategy based on the Baldwin effect is an iterative process of learning and evolution that balances the relationship between global search and local search. To improve the non-dominated solution of particle swarms, an individual particle learns in the same environment to achieve stronger survival adaptability[39].

After completing the Baldwin effect learning operation, the value of a certain dimension of the particle is larger than the value range, so it is necessary to calculate the particle swarm with the extreme value standardization method, which is used if the value is larger than the value range.

Therefore, the value range of each dimension of the particles is a discrete value range $\left\{p_{k}^{j}: 1 \leq p_{k}^{j} \leq K_{j}\right\}$. After the Baldwin effect learning operation is completed, the dimension value of the particles is greater than the value range, and the extreme value normalization method is used for the particle swarm in this excessive value range. If $p_{k}^{j}>K_{j}$, the value is set to $p_{k}^{j}=K_{j}$.

The optimal position of each individual $p_{i}$ of the particle swarm is now $x_{i}=\left(x_{i 1}, x_{i 2}, \ldots, x_{i j}\right)$. The functional generalized derivative $f(x)$ representing the feed- 
back information in the learning and evolution process of the BM-MOPSO algorithm is calculated, which is defined as follows:

$$
\frac{D f\left(p_{i}\right)}{D x_{i k}}=\frac{f\left(x_{i 1}, \ldots, x_{i k}+\Delta x_{i k}, \ldots, x_{i j}\right)-f\left(x_{i 1}, \ldots, x_{i k}, \ldots, x_{i j}\right)}{\Delta x_{i k}}
$$

Here, the individual particle $p_{i}$ yields $p_{i}$ ' when the learning strategy based on the Baldwin effect is carried out. For each dimension $x_{i k}(k=1,2, \ldots, j)$ of the position vector of individual, the formula is as follows:

$$
\begin{aligned}
\min _{\left|p_{i}-p_{i}^{\prime}\right| \leq \varepsilon} f\left(p_{i}^{\prime}\right) & =\min _{\left|p_{i}-p_{i}^{\prime}\right| \leq \varepsilon} f\left(x_{i 1}^{\prime}, x_{i 2}^{\prime}, \ldots, x_{i j}^{\prime}\right) \\
& =\min _{N_{i 1}, N_{i 2}, N_{i j} \in Z^{+}} f\left(x_{i 1}+\frac{\lambda_{i 1} N_{i 1} D f\left(p_{i}\right)}{D x_{i 1}}, \ldots, x_{i j}+\frac{\lambda_{i j} N_{i j} D f\left(p_{i}\right)}{D x_{i j}}\right)^{\prime}
\end{aligned}
$$

where $\lambda_{i k}$ and $N_{i k}$ are the parameters for adjusting the step length. The integer $N_{i k}$ is the number of individuals in the population particle swarm $p_{i}$, and their initial values are all the same; $N_{i k}+1 \rightarrow N_{i k}$ and $\lambda_{i k}$ is the value of the updated particle position within the feasible region $\left[x_{L}, x_{R}\right][40]$. Then, $\lambda_{i}$ is the quotient of the range distance and the maximum iteration number:

$$
\lambda_{i}=\frac{\left|x_{L}-x_{R}\right|}{T_{\max }},
$$

The steps of the algorithm are as follows:

Step 1: Initialization. This includes all parameters of the particles, such as the initial position and velocity. The individual optimal position of a particle is defined as the current position, and the global optimal position is the optimal position of all particles. The initial position of each particle $i$ is $x_{i}=\left(x_{1}, x_{2}\right)$, the speed is $v_{i}=\left(v_{1}, v_{2}\right)(i=1, \ldots, N)$, the number of particles is, the number of iterations is $n_{\max }$, and the initial solutions $p^{0}$ are randomly generated by $n_{p}$.

Step 2: Calculate the fitness function value of each particle. That is, the fitness function value fitness $\left(p^{i}\right)$ is calculated by the functional generalized derivative $f(x)$ when the coordinate $x_{i}$ takes the coordinates into the generalized derivative.

Step 3: Determine whether to perform local search. If the current iteration number meets the conditions for iteration termination $(<1 / 3$ of the total number of iterations), then local search is not performed and the algorithm moves to step 4; otherwise, step 5 is performed.

Step 4: Perform a global search. If the number of consecutive iterations with no updates meets the preset threshold, each particle will calculate the update speed and position in the global optimal solution according to the basic formula; otherwise, the algorithm moves to step 6;

Step 5: Perform a local search. The particles perform the local search based on the Baldwin effect with probability $P_{\mathrm{k}}$, and the algorithm moves to step 6; 
Step 6: Non-uniform mutation. Assuming that there is a particle $p^{t}=\left(v_{1}, \ldots, v_{j}\right)$ in the $t$-th generation, a random variable $v_{\mathrm{k}}$ is selected and outputs a number between 0 and 1 , and a non-uniform mutation operator is locally mutated by the particle for the next generation $p^{t+1}=\left(v_{1}, \ldots, v_{k}^{\prime}, \ldots, v_{j}\right)$. The particle swarm will become more stable.

Step 7: Repeat step 2 to step 6 until the current iteration number meets the conditions for termination; otherwise, continue to update $\mathrm{x}_{i}$ and $v_{i}$.

Step 8: After execution, output the calculation result.

\section{Example simulation}

To ensure the preciseness of the data, the case data were sampled from a CMfg platform developed by a 3D printing company in Wuhan city, which is a smart C3DPS platform that integrates modelling design and 3D printing. It integrates various kinds of C3DPSs of multiple fields and types and provides network access to different types of 3D printing equipment. It also performs online real-time data collection. Here, the real data of different 3D printing devices in the platform were selected for the example simulation.

\subsection{Simulation environment}

To verify the reliability and universality of the cloud service evaluation based on the hybrid multi-objective BM-MOPSO evaluation model, a verification application in C3DPS creative and innovation product development was used. Its simulation environment was as follows:
a. Windows 7 operating system
b. Intel (R) Core (tm) i5-4210H 2.90 GHZ CPU
c. $8 \mathrm{G}$ memory.

The experimental environment was as follows:

At run time, the population size was 10, the maximum number of iterations was 100, the target weights were $Q_{1}$ and $Q_{2}$, and the values were 0.7 and 0.3 , respectively. The range of moderate function deterioration was set to -0.1 for the moderate functions $w_{1}=0.20, w_{2}=0.20, w_{3}=0.10, w_{4}=0.05, \quad w_{5}=0.10, \quad w_{6}=0.20$, $w_{7}=0.05, \quad w_{8}=0.10$.

According to the above multi-objective BM-MOPSO, the evaluation data and weight value of each candidate 3D printing device were inputted and simulated in MATLAB. Each algorithm was tested independently for each test function fewer than 30 times. The convergence characteristics of the algorithms are shown in Figure 4 and Figure 5: 


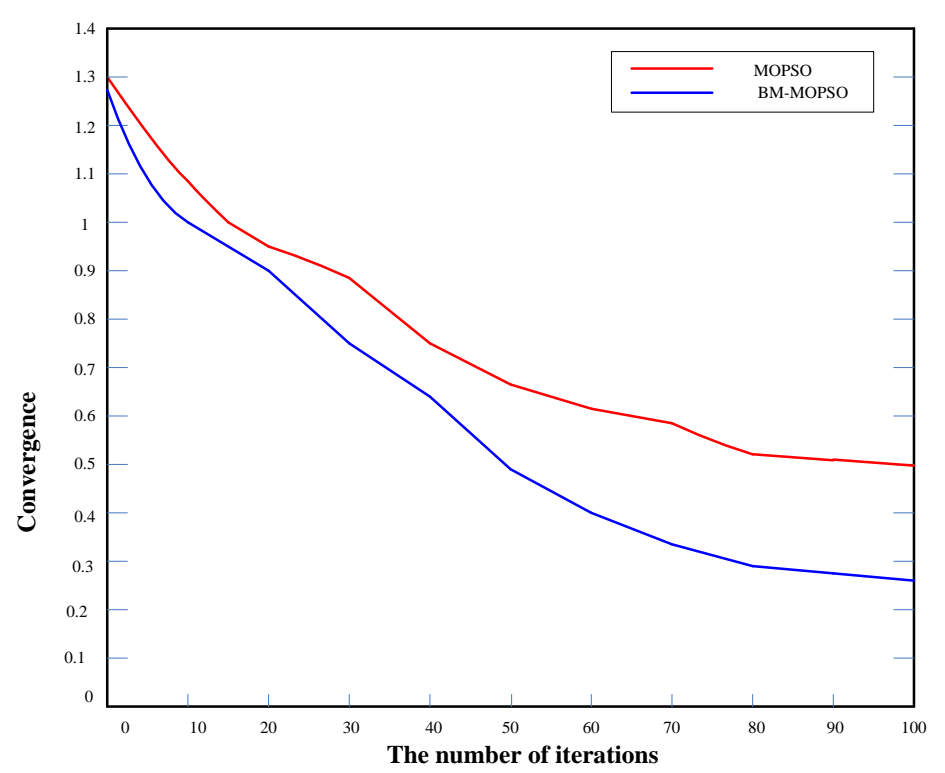

Figure 4. Convergence characteristics.

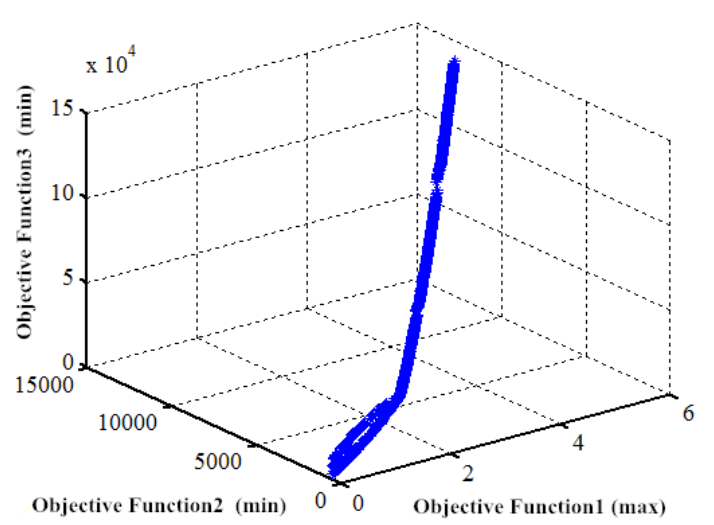

(a)

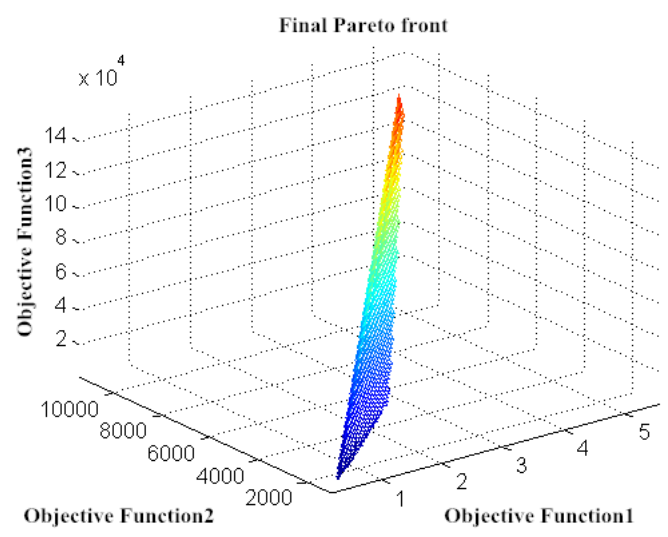

(b)

Figure 5. Simulation results of the algorithm. (a) Iterative simulation of the objective function; (b) The final Pareto frontier based on the improved BM-MOPSO algorithm. A caption on a single line should be centered.

Figure 5. Simulation results of the algorithm.

Flow chart of cloud service evaluation implementation based on the hybrid multi-objective BM-MOPSO evaluation model

\subsection{Analysis of hybrid multi-objective BM-MOPSO}

The Knowledge module is responsible for the management and scheduling of various types of knowledge on service resources; the Coordination module creates links between various coordination methods and performs operation monitoring and coordination management in the cloud service evaluation model based on the hybrid multi-objective BM-MOPSO evaluation model. Figure 6 shows the evaluation process for cloud services based on the hybrid multi-objective BM-MOPSO evaluation model. 


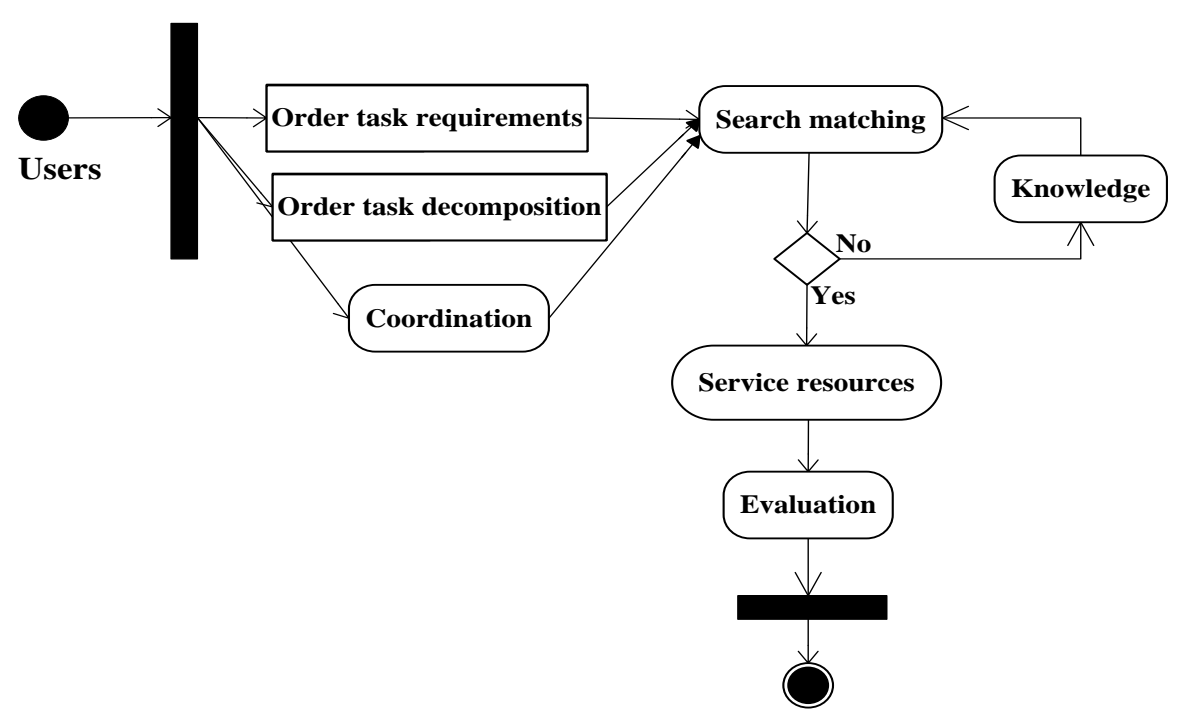

Figure 6. Flow chart of cloud service evaluation implementation based on the hybrid multi-objective BM-MOPS evaluation model.

A user proposes a complex manufacturing task that decomposes into 6 sub-task nodes $T=\left(T_{1}, T_{2}, T_{3}, T_{4}, T_{5}, T_{6}\right)$. After entering the task information into the platform, it is preliminarily matched to several 3D printing equipment resources that meet the user's needs. A candidate set of 3D printing equipment resources is established, that is, $S=\left(S_{1}, S_{2}, S_{3}, S_{4}, S_{5}, S_{6}\right)$. Among them, task node $S_{1}$ is matched with three candidate resources, which can be expressed by $S_{1}=\left(S_{11}, S_{12}, S_{13}\right)$. Each candidate resource is shown in Table 2.

Table 2. Candidates for 3D printing device resources.

\begin{tabular}{cccc}
\hline Candidate set & Atomic service & Workshop name & Equipment model \\
\hline$S_{1}$ & $S_{12}$ & Yourui 3D printing & HW-602 \\
& $S_{13}$ & JIA Yi Gaoke & JOYE-4035 \\
& $S_{21}$ & Campus store & Aurora LVO A8 \\
& $S_{22}$ & WINBO & WB-SH105 \\
$S_{2}$ & $S_{23}$ & Beien 3D & BANSOT M2 \\
& $S_{24}$ & 3D printing workshop & Dimension SST 1200es \\
& $S_{31}$ & The third brother of Hanbang & Corporate T1 \\
& $S_{32}$ & Artful design workshop & SLM 500 \\
$S_{3}$ & $S_{33}$ & E-Plus-3D & EP-M100T \\
& $S_{41}$ & Manheng & EOS-M290
\end{tabular}




\begin{tabular}{|c|c|c|c|}
\hline & $S_{42}$ & Flashcast Technology Studio & Explorer \\
\hline & $S_{43}$ & Yourui 3D printing & DLP-1 \\
\hline \multirow{4}{*}{$S_{5}$} & $S_{51}$ & Flashcast Technology Studio & Creator Pro \\
\hline & $S_{52}$ & Wuhan store & Second-generation 3D printing \\
\hline & $S_{53}$ & JIA Yi Gaoke & JOYE-1010K \\
\hline & $S_{54}$ & JIA Yi Gaoke & JOYE-1212E \\
\hline \multirow{4}{*}{$S_{6}$} & $S_{61}$ & Campus Station of College of Culture & FORTUS 200 mc \\
\hline & $S_{62}$ & 3D printing workshop & ProJet 6000 \\
\hline & $S_{63}$ & Yunle Design Studio & ULTRA \\
\hline & $S_{64}$ & High-precision printing & HOFTX2 \\
\hline
\end{tabular}

According to the different 3D printing equipment resources, the evaluation indicators are optimized and quantified from the original data of each 3D printing equipment resource. The form of the data set is as follows:

$$
S_{1}=\left[\begin{array}{ccccc}
q_{c}\left(S_{i 1}\right) & q_{t}\left(S_{i 1}\right) & q_{r}\left(S_{i 1}\right) & q_{u}\left(S_{i 1}\right) & q_{e}\left(S_{i 1}\right) \\
q_{c}\left(S_{i 2}\right) & q_{t}\left(S_{i 2}\right) & q_{r}\left(S_{i 2}\right) & q_{u}\left(S_{i 2}\right) & q_{e}\left(S_{i 2}\right) \\
q_{c}\left(S_{i 3}\right) & q_{t}\left(S_{i 3}\right) & q_{r}\left(S_{i 3}\right) & q_{u}\left(S_{i 3}\right) & q_{e}\left(S_{i 3}\right) \\
\vdots & \vdots & \vdots & \vdots & \vdots \\
q_{c}\left(S_{i j}\right) & q_{t}\left(S_{i j}\right) & q_{r}\left(S_{i j}\right) & q_{u}\left(S_{i j}\right) & q_{e}\left(S_{i j}\right)
\end{array}\right]
$$

Here, the unit of $q_{c}\left(S_{i j}\right)$ is "Yuan", and the unit of $q_{t}\left(S_{i j}\right)$ is "hour".

The above evaluation data of each candidate 3D printing equipment resource are specifically expressed as follows:

$$
\begin{aligned}
& S_{1}=\left[\begin{array}{lllll}
1240 & 35 & 0.76 & 0.79 & 78 \\
1300 & 55 & 0.18 & 0.48 & 94 \\
1750 & 54 & 0.44 & 0.64 & 98
\end{array}\right] S_{2}=\left[\begin{array}{ccccc}
980 & 42 & 0.79 & 0.75 & 20 \\
2200 & 59 & 0.27 & 0.65 & 34 \\
1700 & 61 & 0.65 & 0.16 & 70 \\
3200 & 59 & 0.11 & 0.49 & 30
\end{array}\right] \\
& S_{3}=\left[\begin{array}{llllll}
1400 & 49 & 0.95 & 0.34 & 53 \\
1300 & 58 & 0.58 & 0.22 & 64 \\
2700 & 67 & 0.75 & 0.25 & 87
\end{array}\right] S_{4}=\left[\begin{array}{lllll}
1850 & 30 & 0.50 & 0.69 & 49 \\
3300 & 34 & 0.89 & 0.95 & 40 \\
2000 & 24 & 0.54 & 0.13 & 68
\end{array}\right]
\end{aligned}
$$




$$
S_{5}=\left[\begin{array}{ccccc}
1600 & 55 & 0.14 & 0.25 & 49 \\
2600 & 36 & 0.84 & 0.25 & 68 \\
1850 & 37 & 0.81 & 0.24 & 75 \\
900 & 44 & 0.92 & 0.35 & 53
\end{array}\right] S_{6}=\left[\begin{array}{ccccc}
750 & 43 & 0.19 & 0.25 & 35 \\
2700 & 37 & 0.61 & 0.47 & 67 \\
1800 & 38 & 0.35 & 0.83 & 59 \\
1000 & 68 & 0.58 & 0.54 & 76
\end{array}\right],
$$

Assuming that the particle population is 14 and the number of iterations is 50 , the weight values of the evaluation parameters in the fitness function are, respectively $\alpha_{1}=0.2, \alpha_{2}=0.3, \alpha_{3}=0.2, \alpha_{4}=0.1$ and $\alpha_{5}=0.2$.

According to the above multi-objective BM-MOPSO, the evaluation data and weight value of each candidate 3D printing device are inputted and simulated in MATLAB. The simulation results are shown in Figure 7, in which the vertical axis is the fitness function value and the horizontal axis is the population number.

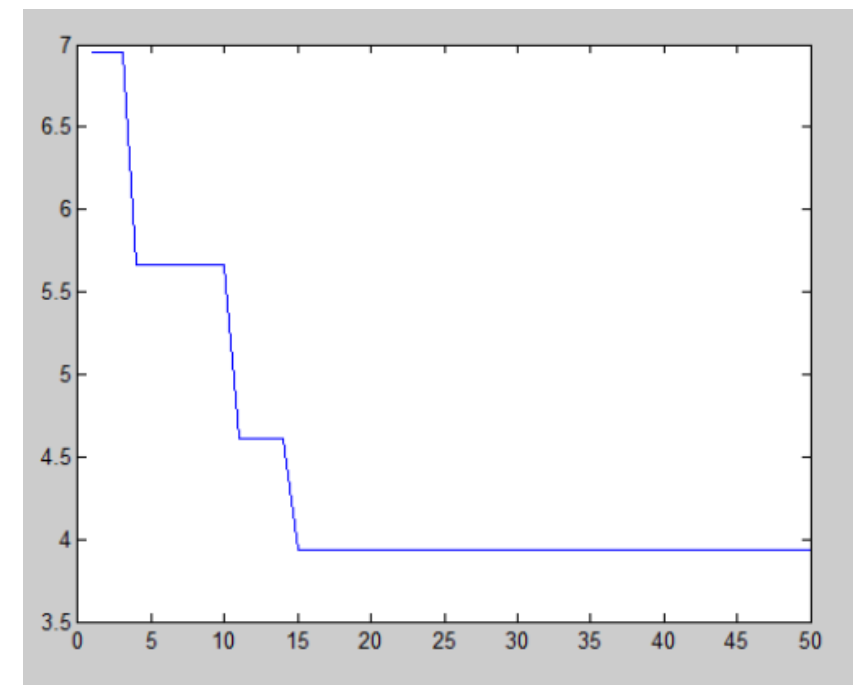

Figure 7. Convergence curve of the fitness function.

It can be seen from Fig. 6 that when the population number is 14, the fitness is lowest, the optimal combination scheme is, and the fitness function value is 3.9370 . That is, the optimal combination of 3D printing equipment resources is Yourui 3D printing hw-602, WINBO WB-SH105 and Qiaoyi workshop SLM 500, Flashcast Technology Explorer, Jiayi Hi-Tech JOYE-1212E, and Yunle Design Studio.

\section{Conclusions}

The global economy is transforming from a product economy to a service economy. Manufacturing and services are gradually merging. Individual enterprises pay close attention to their own core business. By providing manufacturing services, they can increase the value for stakeholders in the manufacturing value chain so that these individual enterprises will be more closely coordinated with each other. The C3DP model is a new service model that supports multi-user collaboration, and it is also an application of cloud manufacturing in the field of 3D printing. C3DPS modelling is the basis of C3DPS supply and demand matching; that is, this relationship between order tasks and services provides an effective way to match similar elements in the C3DPS platform. A large number of C3DPSs are aggregated according to certain rules and form a multi-complex $3 \mathrm{D}$ printing service network.

This paper formally describes C3DPSs, proposes a QoS acquisition and calculation method based on a mutual evaluation mechanism under the CMfg model, and establishes a C3DPS trust evaluation model based on service matching and global trust. The 
genetic algorithm optimizes the combination of C3DPSs so that it meets the requirements. Active intelligent rent-seeking for C3DPSs will be the next research direction.

\begin{abstract}
Author Contributions:
Conceptualization, Z.C.L. and Z.C.S.; methodology, Z.C.L.; software, L.J.J. And Z.J.J; validation, H.H. and Y.B.; formal analysis, Z.C.L. and L.J.J.; investigation, Z.C.L., L.J.J., and Z.S.L.; resources, L.R.L.; data curation, Z.C.L; writing - original draft preparation, Z.C.L. and L.J.J; writing - review and editing, Z.C.S. ; visualization, Z.J.J. and H.H.; supervision, Z.C.S. and L.R.L.; project administration, L.R.L.; funding acquisition, H.H. All authors have read and agreed to the published version of the manuscript.
\end{abstract}

Funding: This work is supported by the Natural Science Foundation of Shan dong Province of China under Grant (ZR2019PEE019) and High-level talents (high-level doctorate) research project of Linyi University (LYDX2019BS009).

Informed Consent Statement: Not applicable.

Data Availability Statement: The data presented in this study are available on request from the first author.

Acknowledgments: We sincerely thank Garrett Chapman Goble for his linguistic assistance during the preparation of this manuscript. We are grateful to the editors and anonymous reviewers for the valuable comments and suggestions on the manuscript.

Conflicts of Interest: The authors declare no conflict of interest.

\title{
References
}

1. Ouelhadj, D., Petrovic, S., A Survey of Dynamic Scheduling in Manufacturing Systems. Journal of Scheduling. 2008, 12, 4, 417-431.doi:10.1007/s10951-008-0090-8.

2. Wong, T. N., Leung, C. W., Mak, K. L., Fung, R. Y. K. 2006., Dynamic Shopfloor Scheduling in Multi-Agent Manufacturing Systems. Expert Systems with Applications. 2006, 31, 3, 486-494. doi:10.1016/j.eswa.2005.09.073.

3. Xiang, W., Lee, H. P.,Ant Colony Intelligence in Multi-Agent Dynamic Manufacturing Scheduling. Engineering Applications of Artificial Intelligence. 2008, 21, 1, 73-85. doi:10.1016/j.engappai.2007.03.008.

4. Rahman,I. U., Zakarya, M., Raza, M., et al. An n-state switching PSO algorithm for scalable optimization[J]. Soft Computing. 2020, 11, 11297-11314.

5. Nie, L., Gao, L., Li, P., Shao, X. Reactive Scheduling in a Job Shop Where Jobs Arrive over Time. Computers and Industrial Engineering. 2013, 66, 2, 389-405. doi:10.1016/j.cie.2013.05.023.

6. Zeng, N., Wang, Z., Li, Y., et al. A Hybrid EKF and Switching PSO Algorithm for Joint State and Parameter Estimation of Lateral Flow Immunoassay Models. IEEE/ACM Transactions on Computational Biology \& Bioinformatics. 2012, 9 , 2, 321-329.

7. P.Cowling, M. Johansson.,Using Real Time Information for Effective Dynamic Scheduling. European Journal of Operational Research. 2002, 139, 2, 230-244. doi:10.1016/S0377-2217(01)00355-1.

8. Hwang., H. C., B. K. Choi. Workflow-Based Dynamic Scheduling of Job Shop Operations. International Journal of Computer Integrated Manufacturing. 2007, 20, 6, 557-566. doi:10.1080/09511920601024179.

9. Kim, S., Lee, J., Choi, B. 3D printed fluidic valves for remote operation via external magnetic field. International Journal of Precision Engineering and Manufacturing. 2016, 17, 7, 937-942.

10. Guerrero, G., Langa, J. A., Suárez, A. Architecture of attractor determines dynamics on mutualistic complex networks. Nonlinear Analysis Real World Applications. 2017, 34, 17-40.

11. Ikram, M., Sroufe, R., Zhang, Q. Prioritizing and overcoming barriers to integrated management system (IMS) implementation using AHP and G-TOPSIS. Journal of Cleaner Production, 2020, 254, 1-17. DOI: 10.1016/j.jclepro.2020.120121.

12. Fatima, A., Cyril, G., Vincent, V., et al. Towards normalization selection of Raman data in the context of protein glycation: application of validity indices to PCA processed spectra. Analyst. 2020, 145, 1-12.DOI:10.1039/C9AN02155H. 
13. Cheng, Y., Tao, F., Zhang, L., Zhao, D. Dynamic Supply-Demand Matching for Manufacturing Resource Services in Service-Oriented Manufacturing Systems: A Hypernetwork-Based Solution Framework. ASME 2015 International Manufacturing Science and Engineering Conference. 2015, 429, V002T04A017-1; 7.

14. Aslam, M., Fahmi, A., Almahdi, F. A. A., et al. Extension of TOPSIS method for group decision-making under triangular linguistic neutrosophic cubic sets. Soft Computing. 2021, 25, 5, 3359-3376.

15. Tao, F., Cheng, J., Cheng, Y., et al. SDMSim: A manufacturing service supply-demand matching simulator under cloud environment. Robotics \& Computer Integrated Manufacturing. 2017, 45, 6, 34-46.

16. Xia, B., Liu, T., Ding, T., et al. An Improved PSO Localization Algorithm for UWB Sensor Networks. Wireless Personal Communications. $2020,15,1-17$.

17. Cheng, Y., Tao, F., Lu, X., et al. Advanced manufacturing systems: supply-demand matching of manufacturing resource based on complex networks and Internet of Things. Enterprise Information Systems. 2016, 2016, 1-18.

18. Cheng, Y., Tao, F., Zhao, D., et al. Modeling of manufacturing service supply-demand matching hypernetwork in service-oriented manufacturing systems. Robotics \& Computer Integrated Manufacturing. 2016, 45, 59-72.

19. Raman, M. R., Somu, N., Kirthivasan, K., et al., A Hypergraph and Arithmetic Residue-based Probabilistic Neural Network for classification in Intrusion Detection Systems. Neural Networks. 2017, 92, 52-64.

20. Sheng, B. Y., Zhao, F. Y., Zhang, C.L., et al. 3D Rubik's Cube-online 3D modeling system based on Web GL. Technology, Networking, Electronic \& Automation Control Conference. IEEE. 2018. DOI: 10.1109/ITNEC. 2017.8284798.

21. Kim, J. H., Song, H. Y. Hypergraph-based Binary Locally Repairable Codes with Availability. IEEE Communications Letters. 2017, 99, 1-1.

22. Zhang, C. L., Sheng, B. Y., Yin, X. Y., et al. Research and development of off-line services for the 3D automatic printing machine based on cloud manufacturing. Journal of Ambient Intelligence \& Humanized Computing. 2017, 1, 1-20.

23. MN, A., D, A. A., R, B. M. Optimum fuzzy sliding mode control of fuel sloshing in a spacecraft using PSO algorithm. Acta Astronautica. 2020, 167, 331-342.

24. Minguella, J., Villegas, M., Poll, B., et al. Automatic Casting of Advanced Technical Ceramic Parts via Open Source High Resolution 3D Printing Machines. Key Engineering Materials. 2015, 631, 269-274.

25. Im, S., Lee, Y., Kim, J., et al. A solution for camera occlusion using a repaired pattern from a projector. International Journal of Precision Engineering \& Manufacturing. 2016, 17, 11, 1443-1450.

26. Yasser, Y. A new knowledge-based link recommendation approach using a non-parametric multilayer model of dynamic complex networks. Knowledge-Based Systems. 2018, 143, 81-92.

27. Wang, X. G., Sheng, B. Y., Zhang, C. L., et al. An effective application of 3D cloud printing service quality evaluation in BM-MOPSO. Concurrency \& Computation Practice \& Experience. 2018. DOI: https://doi.org/ 10.1002 /cpe.4977.

28. Yasser, Y., Farshad, S. A statistical infinite feature cascade-based approach to anomaly detection for dynamic social networks. Knowledge-Based Systems. 2017, 100, 52-64.

29. Tao, F., Zhang, L., Lu, K., Zhao, D. Study on manufacturing grid resource service optimal-selection and composition framework. Enterp Inf Syst. 2012, 6, 2, 237-264.

30. Tao, F., Li, C., Liao, T. W., Laili, Y. J. BGM-BLA: a new algorithm for dynamic migration of virtual machines in cloud computing. IEEE Transactions on Services Computing. 2016, 99, 910-925.

31. Zhang, C. L., Liu, J. J., Xu, B., Yuan, B., Zhuang, S. L., Zhao, F. Y. Architecture of cloud 3D printing task modeling for nodes dynamic scheduling and coupling based on complex networks. IEEE Access. 2020, 8, 99, 135208-135222.

32. Ahmed, N. M., Chen, L. An efficient algorithm for link prediction in temporal uncertain social networks. Inf. Sci. 2016, 5, 331, 120-136.

33. Chen, Z., Hendrix, W., Samatova, N.F. Community-based anomaly detection in evolutionary networks. Intell. Inf. Syst. 2012, 5, 39, No, 159-85.

34. Fanaee, T. H., Gamab, J. Tensor-based anomaly detection: An interdisciplinary survey. Knowl.-Based Syst. 2016, 5, 98, $130-147$. 
35. Heard, N. A., Weston, D. J., Platanioti, K., Hand, D. J. Bayesian anomaly detection methods for social networks. Ann. Appl. Stat. Inst. Math. Stat. 2016, 5, 4, 2, 645-662.

36. Anwar, H., Din, I., Kang, P. Projector calibration for 3D scanning using virtual target images. International Journal of Precision Engineering and Manufacturing. 2012, 13, 1, 125-131.

37. Zhang, C. L., Zhao, F. Y., Wang, Z. Q. Modeling of Cloud 3D printing service hyper-network in service-oriented manufacturing systems. IEEE Access. 2019, 8, 99, 16225-16235.

38. Zhou, L., Zhang, L., Laili, Y., et al. Multi-task scheduling of distributed 3D printing services in cloud manufacturing. International Journal of Advanced Manufacturing Technology. 2018, 96, 2, 3003-3017.

39. Yong, B. S., Yun, Y. H., Joo, K. N. 3D multi-layered film thickness profile measurements based on photometric type imaging ellipsometry. International Journal of Precision Engineering and Manufacturing. 2016, 17, 8, 989-993.

40. Liu, C., Cao, G. H., Yong-Yin, Q. U., et al. An improved PSO algorithm for time-optimal trajectory planning of Delta robot in intelligent packaging. International Journal of Advanced Manufacturing Technology. 2020, 107, 1, 1091-1099. 